\title{
Compétences linguistiques version «light»
}

\section{Christoph Hänggeli}

Avocat, directeur de I'ISFM

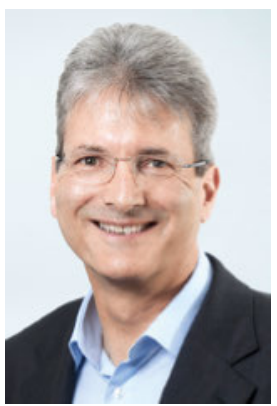

Souvenons-nous [1]: le 20 mars 2015, les Chambres fédérales ont définitivement approuvé la révision de la loi sur les professions médicales universitaires (LPMéd). Grâce au soutien des autres professions réglementées dans la LPMéd (Société suisse des médecins-dentistes SSO, Chirosuisse, pharmaSuisse et Société des vétérinaires suisses SVS), la FMH et l'ISFM sont parvenus à faire inscrire dans la loi deux aspects importants de la protection des patients. Dès 2018, toutes les personnes qui souhaitent exercer une profession médicale à titre salarié ou indépendant en Suisse devront remplir les conditions suivantes:

- figurer dans le registre officiel des professions médicales, inscription qui garantit notamment la vérification officielle des diplômes étrangers;

- justifier les connaissances linguistiques nécessaires à l'exercice de leur profession.

La concrétisation et la mise en œuvre des connaissances linguistiques minimales exigées relèvent de la compétence du Conseil fédéral. A cet effet, l'Office fédéral de la santé publique (OFSP) a élaboré une ordonnance d'application et mené une procédure de consultation à large échelle. Aucune réglementation efficace n'est en vue pour garantir les compétences linguistiques. Si l'office fédéral envisage bien d'inscrire dans le registre toutes les connaissances linguistiques attestées, p. ex. l'anglais pour un médecin originaire des Etats-Unis et le croate pour un médecin originaire de Croatie, le projet d'ordonnance renonce en revanche à exiger des médecins salariés la maîtrise d'une langue

\section{Avant de débuter une activité en Suisse, tous les médecins étrangers devront à l'avenir faire reconnaître ou vérifier leur diplôme.}

nationale suisse. Soucieux d'une bonne communication médecin-patient, nous avons donc proposé lors de la consultation d'inscrire au minimum des connaissances d'une langue officielle suisse de niveau B2 dans l'ordonnance sur les professions médicales; le niveau B2 se fonde sur le Cadre européen commun de référence pour les langues et correspond à peu près au niveau maturité.
Cette revendication est encore bien en-deçà des standards appliqués dans d'autres pays européens. L'Allemagne et l'Autriche, par exemple, organisent des tests de langue approfondis comprenant notamment une simulation d'entretien médecin-patient qui permet de tester les connaissances actives et passives du vocabulaire technique. Ces tests affichent un taux d'échec de $30 \%$. Dans ces pays, tout le monde s'accorde à dire que les médecins doivent pouvoir communiquer avec aisance dans une langue nationale non seulement avec les patients mais aussi avec leurs collègues afin d'éviter toute erreur de diagnostic et de traitement en raison d'une mauvaise compréhension.

Rien de tel en Suisse: ici, on invoque des arguments comme «il faut laisser faire le marché» ou «les hôpitaux peuvent envoyer leurs nouvelles recrues suivre des

Dans d'autres pays européens, la profession de médecin ne peut pas être exercée sans la maîtrise d'une langue nationale.

cours de langue intensifs». Or dans un secteur aussi sensible que celui de la santé, des lacunes linguistiques peuvent rapidement avoir des conséquences dramatiques et mettre des vies en jeu. Et pas seulement: la Suisse doit-elle rester un îlot au milieu de l'Europe au risque de créer un appel d'air pour tous les médecins qui sont empêchés de pratiquer dans les pays voisins en raison de connaissances linguistiques insuffisantes?

Le Conseil fédéral devrait se prononcer ce mois encore sur la teneur définitive de l'ordonnance sur les professions médicales. Décidera-t-il de suivre la proposition de la FMH / de l'ISFM en exigeant des connaissances d'une langue officielle de niveau B2 minimum dans l'intérêt de la sécurité des patients et de la qualité des soins? Ou la Suisse restera-t-elle la lanterne rouge en Europe avec les exigences linguistiques les plus faibles?

\section{Référence}

1 Schlup J, Hänggeli C. Révision de la LPMéd: tout est bien qui finit bien? Bull Méd Suisse. 2015;96(19):677. 